\title{
Effect of Bariatric Surgery on Anthropometric and Biochemical Parameters in Morbidly Obese Patients
}

\author{
Ashish Ahuja ${ }^{1}$, Jagdeep Choudhary ${ }^{2}$, Preeti Bajaj ${ }^{3}$
}

\begin{abstract}
Background: The aim of this study was to evaluate the effect of bariatric surgery on the anthropometric and biochemical parameters of patients. The effect of surgery on anthropometric parameters like weight, body mass index (BMI), waist circumference, hip circumference, and waist-hip ratio was studied. The biochemical parameters included glycated hemoglobin $A(\mathrm{HbA} 1 \mathrm{c})$ and lipid profile [serum cholesterol, triglycerides, high-density lipoprotein (HDL), low-density lipoprotein (LDL), and very low-density lipoprotein (VLDL)].

Materials and methods: The study was conducted in a tertiary healthcare center in a 1.5-year period on male obese subjects who had undergone bariatric surgery for morbid obesity. Thorough preoperative evaluation was done by a bariatric team which included the bariatric surgeon, dietician, endocrinologist, chest physician, anesthesiologist, and cardiologist. A total of 17 patients who underwent surgery for morbid obesity and gave consent were included in the study and were followed up for 3 months thereafter.

Results: Significant weight loss along with a significant decrease in BMI, waist circumference, hip circumference, and waist-hip ratio was observed at 4 weeks and 3 months after bariatric surgery. A statistically significant difference was seen in the fall in HbA1c levels. There was a significant effect on serum cholesterol, serum triglycerides, and serum HDL and LDL levels. No statistically significant difference was seen in serum VLDL levels.

Conclusion: Significant weight loss after bariatric surgery in men results in improved clinical outcomes. Lipid profile and glycemic control also improved in patients over follow-up time. The improvement in metabolic parameters may serve as motivators for obese men considering surgery. Keywords: Anthropometry, Bariatric surgery, Glycated hemoglobin A, Morbid, Obesity, Weight loss.

World Journal of Laparoscopic Surgery (2019): 10.5005/jp-journals-10033-1386
\end{abstract}

\section{INTRODUCTION}

Obesity is a worldwide epidemic. It is one of the leading preventable causes of death all over the world with an increasing prevalence in both adults and children. It is one of the most serious public health problems of the 21 st century. ${ }^{1}$

Globally, there are more than 1 billion adults who are overweight with at least 300 of them being obese. Obesity is now recognized as a "disease" because it is a physiologic dysfunction of the human organism with environmental, genetic, and endocrinological causes.

Obesity most commonly develops when the caloric intake of food exceeds energy expenditure over a prolonged period of time. Factors influencing obesity involve energy intake or expenditure (or both) and are affected by genetic, behavioral, cultural, along with socioeconomic factors. Obesity is calculated as Quetelet's body mass index (BMI) which is a ratio of weight (measured in kilograms) to height (measured in square meters). Persons with $\mathrm{BMI}>30 \mathrm{~kg} / \mathrm{m}^{2}$ are considered as obese. Morbid obesity (BMI $>40 \mathrm{~kg} / \mathrm{m}^{2}$ ) is the harbinger of many diseases that affect essentially every organ system like cardiovascular, respiratory, metabolic, musculoskeletal, endocrinal, reproductive, dermatological, neurological, and many more. ${ }^{2}$ Bariatric surgery has been recognized as the most effective treatment for morbid obesity. ${ }^{3}$

Various options which are available for the treatment of obesity can be broadly categorized into nonsurgical management and surgical interventions. Nonsurgical management of obesity includes behavioral modifications and interventions, pharmacotherapy for weight loss, dietary and herbal medications, and implanted electrical stimulators.

\footnotetext{
${ }^{1}$ Department of Surgery, Dayanand Medical College and Hospital, Ludhiana, Punjab, India

${ }^{2}$ Department of Surgery, PCMS-I, Sub Divisional Hospital, Balachaur, Punjab, India; Dayanand Medical College and Hospital, Ludhiana, Punjab, India

${ }^{3}$ Department of Pathology, Dr Vasantrao Pawar Medical College Hospital and Research Center, Nashik, Maharashtra, India

Corresponding Author: Preeti Bajaj, Department of Pathology, Dr Vasantrao Pawar Medical College Hospital and Research Centre, Nashik, Maharashtra, India, Phone: +91 7350659659, e-mail: dr. prbajaj@gmail.com

How to cite this article: Ahuja A, Choudhary J, Bajaj P. Effect of Bariatric Surgery on Anthropometric and Biochemical Parameters in Morbidly Obese Patients. World J Lap Surg 2019;12(3):96-100.

Source of support: Nil

Conflict of interest: None
}

However, surgical procedures are currently the most effective therapy for long-term weight loss. ${ }^{4}$ In clinical trials, long-term survival is better in the surgically treated group than in those managed conservatively. Furthermore, some of these operations lead to the rapid remission of type II diabetes in a weight loss independent manner. ${ }^{5}$

The number of bariatric procedures performed in the United States increased from 13,365 in 1998 to more than 200,000 in $2008 .^{6}$ Bariatric surgery is usually considered when other weight loss efforts have failed. National Institutes of Health Consensus Development Conference Panel established the eligibility criteria in 1991. These still remain the most widely accepted criteria. Selection and exclusion criteria are listed in Table $1 .^{7-10}$ 
Table 1: Selection and exclusion criteria for bariatric surgery

\section{Selection criteria}

Able to adhere to postoperative care (e.g., follow-up visits and tests, medical management, and use of dietary supplements)

$\mathrm{BMI} \geq 40 \mathrm{~kg} / \mathrm{m}^{2}$

$\mathrm{BMI} \geq 35 \mathrm{~kg} / \mathrm{m}^{2}$ with obesity-related comorbidity

Previous failed nonsurgical attempts at weight reduction, including nonprofessional programs (e.g., weight watchers)

Exclusion criteria

Cardiopulmonary disease that would make the risk prohibitive

Current drug or alcohol abuse

Lack of comprehension of risks, benefits, expected outcomes, alternatives, and required lifestyle changes

Reversible endocrine or other disorders that can cause obesity

Uncontrolled severe psychiatric illness

The current surgical options can be broadly classified as gastric restrictive, malabsorptive procedures, or a combination of these two. ${ }^{11}$ Jejunoileal bypass is the archetype malabsorptive procedure but has been largely abandoned because of profound adverse metabolic consequences that include renal calculi, vitamin deficiency, hypokalemia, hepatic dysfunction, and osteoporosis. ${ }^{12}$ Bariatric surgery has been shown to decrease fat mass in various studies that measured body composition after bariatric surgery. ${ }^{13}$ There is a decrease in both subcutaneous and visceral fat after bariatric surgery. ${ }^{14}$

Three procedures are commonly done: (i) laparoscopic adjustable gastric banding (LAGB), (ii) laparoscopic sleeve gastrectomy (LSG), and (iii) Roux-en-Y gastric bypass (RYGB). In LAGB, a hollow, flexible silicone band is placed around the upper stomach, which causes a restrictive effect thereby reducing stomach capacity and, henceforth, causes rapid feelings of satiety. The band is tightened by injecting saline into the band via a subcutaneous port which is located just inferior to the sternum or lateral to the umbilicus.

The LSG procedure resects most of the body and all of the fundus of the stomach, creating a long, narrow, tubular stomach. This procedure was first used as an initial step before a malabsorptive procedure in very high-risk patients but is now approved as a primary stand-alone procedure. ${ }^{15,16}$

In RYGB, a small gastric pouch is formed by dividing the upper stomach and joining it with the resected end of jejunum, so that food bypasses the stomach and upper small bowel, thereby restricting the size of the stomach and causing some malabsorption. Roux-en-Y gastric bypass may be a better choice in more obese patients and in those with type II diabetes [RYGB is the most common procedure (51\%) performed in the United States and Canada, followed by LAGB (44\%)]..$^{17}$ The biliopancreatic diversion, with or without duodenal switch, is an older procedure that is no longer commonly performed. ${ }^{6}$

The choice of procedure depends on the expertise of the surgeon and surgical center, patient preference, and risk stratification. Several studies have shown that the risk of serious complications decreases with increasing procedure volume of the surgeon and center. ${ }^{18-21}$

\section{Aims and Objectives}

The aim of this study was to study the effect of bariatric surgery on the anthropometric and biochemical parameters of patients.
The effect of surgery on anthropometric parameters like weight, BMI, waist circumference, hip circumference, and waist-hip ratio was studied. The biochemical parameters included $\mathrm{HbA1c}$ and lipid profile (serum cholesterol, triglycerides, HDL, LDL, and VLDL).

\section{Materials and Methods}

\section{Preoperative Evaluation}

The study was carried out in the Department of Surgery, Dayanand Medical College and Hospital, Ludhiana, on male obese subjects who underwent bariatric surgery for morbid obesity. After due institutional ethics committee approval, the study was conducted in a 1.5-year time period. Preoperative evaluation was done by a dedicated bariatric team which included the bariatric surgeon, dietician, endocrinologist, gastroenterologist, respiratory medicine physician, psychiatrist, anesthesiologist, and cardiologist.

The preoperative preparation of the patient included the following:

\section{Counseling}

All patients were counseled regarding:

- Management options available for obesity, including diet, exercise, pharmacotherapy, and surgery.

- Expected weight loss and benefits in terms of sustained weight loss and resolution/improvement of comorbidities.

- General information regarding all available surgical options and risks of surgery including irreversibility of the procedure.

- Need for regular follow-up and strict compliance with the dietary, exercise, and lifestyle modifications advised before and after the surgery.

\section{Nutritional Counseling}

Preoperative and postoperative diet was planned in consultation with the dietician. The target weight was calculated in that session.

\section{Detailed Patient Interview to be Included}

Specific inclusion and exclusion criteria and appropriate statistical methods and tests were applied (Table 1).

Patient's complete medical history including history of diabetes mellitus (DM), hypertension, hypothyroidism, and other comorbidities along with details of treatment, duration, and the dosages of medicines was obtained.

- History of sleep apnea and snoring, including requirement for assisted ventilation, home-based oxygen therapy.

All patients underwent a comprehensive multidisciplinary bariatric evaluation, which included the following:

- Cardiology evaluation: electrocardiogram, echocardiography, and, if required, stress thallium.

- Gastroenterology evaluation: an upper gastrointestinal endoscopy was done to rule out reflux esophagitis, hiatus hernia, and gastric ulcers.

- Evaluation by pulmonary physician included pulmonary function tests and arterial blood gas analysis.

- Endocrinology evaluation for detection, assessment and management of diabetes and hypothyroidism.

Out of a total of 17 patients, 12 (70.59\%) underwent laparoscopic sleeve vertical gastrectomy (LSVG). Mini gastric bypass (MGB) was performed in 4 (23.53\%) and open sleeve gastrectomy (OSG) in 
1 patient (5.88\%) (Fig. 1). Thepatients were kept on a follow-up for 3 months from the date of surgery. Particulars and demographic data of each patient were duly noted including their name, age, sex, occupation, admission number, occupation, and address for any future correspondence (Table 2).

The detailed history of each patient was obtained including history of presenting symptoms, any preexisting comorbid conditions, and the patient's past history including treatment and surgical history.

General physical examination and a thorough systemic examination of all patients were carried out at the time of admission. All the necessary routine preliminary investigations were done. All patients underwent specific anthropometric measurements such as weight, BMI, waist circumference, hip circumference, and waist-hip

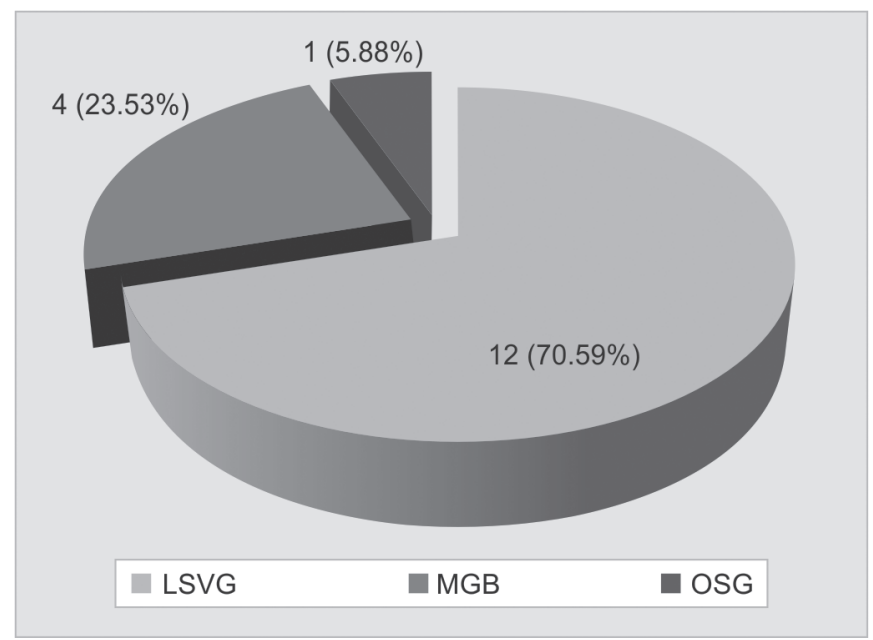

Fig. 1: Distribution of patients on the basis of procedure done

Table 2: Demographic data

\begin{tabular}{ll}
\hline Number $(n)$ & 17 \\
Mean age $($ years $)$ & $43 \pm 7.624$ \\
Mean height $(\mathrm{m})$ & $172.47 \pm 6.09$ \\
Mean weight $(\mathrm{kg})$ & $138.71 \pm 17.51$ \\
Mean BMI $\left(\mathrm{kg} / \mathrm{m}^{2}\right)$ & $46.67 \pm 6.230$ \\
\hline
\end{tabular}

ratio, which were noted preoperatively and at 4 weeks and 3 months postoperatively. The laboratory investigations included $\mathrm{HbA} 1 \mathrm{c}$ and lipid profile, e.g., serum triglycerides, serum cholesterol, serum HDL, serum LDL, and serum VLDL.

Patients presenting included in the study underwent hematological, biochemical, and radiological investigations. For this, patients included in the study were informed regarding their inclusion, and a written informed consent was obtained from all the patients included in the study.

\section{RESULTS}

\section{Effect on Anthropometric Parameters}

Weight was measured before surgery, at 4 weeks, and 3 months after surgery as depicted in Table 3. Preoperatively mean weight was $138.71 \pm 17.51 \mathrm{~kg}$. Significant weight loss was observed at 4 weeks and 3 months when mean weight was $130.47 \pm 14.77 \mathrm{~kg}$ and $116.53 \pm 15.64 \mathrm{~kg}$, respectively, thus concluding that the procedure of bariatric surgery results in effective weight loss.

Body mass index is calculated by weight (in $\mathrm{kg}$ ) divided by height (in $\mathrm{m}^{2}$ ). The patient becomes eligible for bariatric surgery depending upon this main parameter. There was a marked decrease in BMI of the patients at 4 weeks and 3 months postoperatively that was highly statistically significant.

The waist circumference decreased from mean preoperative $132.94 \mathrm{~cm}$ to $126.47 \mathrm{~cm}$ at 4 weeks and to $106.93 \mathrm{~cm}$ at 3 months. The mean hip circumference decreased from preoperative 124.50 $\mathrm{cm}$ to $119.14 \mathrm{~cm}$ at 4 weeks and $108.21 \mathrm{~cm}$ at 3 months. The waist:hip ratio decreased from preoperative $1.06 \mathrm{~cm}$ to $1.05 \mathrm{~cm}$ at 4 weeks and $0.98 \mathrm{~cm}$ at 3 months.

\section{Effect on Biochemical Parameters}

There was a fall in HbA1c levels in patients (mean: $7.14 \pm 2.62$ ) following bariatric surgery (Table 3 ). The fall in $\mathrm{HbA} 1 \mathrm{c}$ levels at 4 weeks (mean: $6.71 \pm 2.22$ ) and 3 months (mean: $5.57 \pm 1.18$ ) postoperatively was significant in both the groups. Serum cholesterol levels showed a significant improvement $(p=0.003)$ at 4 weeks (mean: $209.47 \pm 55.67 \mathrm{mg} / \mathrm{dL}$ ) when compared with preoperative levels (mean: $194.71 \pm 47.17 \mathrm{mg} / \mathrm{dL}$ ) and a highly significant improvement $(p=0.00)$ at 3 months (mean: $169.65 \pm$ $31.06 \mathrm{mg} / \mathrm{dL}$ ). Serum triglyceride levels showed an improvement

Table 3: Value of various parameters before operation and after 4 weeks and 3 months of operation

\begin{tabular}{|c|c|c|c|c|c|c|c|c|}
\hline \multirow[b]{2}{*}{ Parameters } & \multicolumn{2}{|c|}{ Preoperative } & \multicolumn{2}{|c|}{4 weeks } & \multicolumn{2}{|c|}{3 months } & \multirow{2}{*}{$\begin{array}{l}\text { Preoperative } \\
\text { vs } 4 \text { weeks } \\
\text { p value }\end{array}$} & \multirow{2}{*}{$\begin{array}{l}\text { Preoperative } \\
\text { vs } 3 \text { months } \\
\text { p value }\end{array}$} \\
\hline & Mean & $S D$ & Mean & $S D$ & Mean & $S D$ & & \\
\hline Weight (kg) & 138.71 & 17.510 & 130.47 & 14.770 & 116.53 & 15.649 & 0.000 & 0.000 \\
\hline Weight loss & - & - & 8.47 & 3.68 & 22.29 & 4.91 & & \\
\hline$\%$ EWL & - & - & 12.96 & 4.04 & 36.11 & 8.99 & & \\
\hline Waist circumference & 132.94 & 13.64 & 126.47 & 13.24 & 106.93 & 10.83 & 0.170 & 0.000 \\
\hline Hip circumference & 124.50 & 8.28 & 119.14 & 7.96 & 108.21 & 7.48 & 0.063 & 0.000 \\
\hline Waist:hip ratio & 1.06 & 0.06 & 1.05 & 0.06 & 0.98 & 0.05 & 0.630 & 0.002 \\
\hline BMI & 46.67 & 6.230 & 43.95 & 5.317 & 38.67 & 4.610 & 0.000 & 0.000 \\
\hline $\mathrm{HbA1c}$ & 7.14 & 2.628 & 6.71 & 2.222 & 5.57 & 1.181 & 0.008 & 0.002 \\
\hline Serum cholesterol & 209.47 & 55.679 & 194.71 & 47.170 & 169.65 & 31.068 & 0.003 & 0.000 \\
\hline Triglyceride & 305.47 & 76.81 & 287.70 & 70.87 & 250.88 & 63.08 & 0.488 & 0.030 \\
\hline $\mathrm{HDL}$ & 25.23 & 5.70 & 29 & 5.91 & 38.94 & 8.16 & 0.067 & 0.0001 \\
\hline LDL & 99.47 & 40.88 & 94.41 & 38.23 & 86 & 34.08 & 0.711 & 0.304 \\
\hline VLDL & 37.23 & 15.91 & 35.82 & 14.79 & 32.47 & 11.87 & 0.790 & 0.330 \\
\hline
\end{tabular}

$\mathrm{SD}$, standard deviation 
( $p=0.488$ ) at 4 weeks (mean: $287.70 \pm 70.87 \mathrm{mg} / \mathrm{dL}$ ) when compared with preoperative levels (mean: $305.47 \pm 76.81 \mathrm{mg} / \mathrm{dL}$ ) and a significant improvement $(p=0.030)$ at 3 months (mean: $250.88 \pm 63.08 \mathrm{mg} / \mathrm{dL}$ ). Serum HDL levels showed an improvement ( $p=0.067$ ) at 4 weeks (mean: $29 \pm 5.91 \mathrm{mg} / \mathrm{dL}$ ) when compared with preoperative levels (mean: $25.23 \pm 5.70 \mathrm{mg} / \mathrm{dL}$ ) and a highly significant improvement ( $p=0.0001$ ) at 3 months (mean: $38.94 \pm$ $8.16 \mathrm{mg} / \mathrm{dL}$ ). Serum LDL levels showed a decreasing trend ( $p=0.711$ ) at 4 weeks (mean: $94.41 \pm 38.23 \mathrm{mg} / \mathrm{dL}$ ) when compared with preoperative levels (mean: $99.47 \pm 40.88 \mathrm{mg} / \mathrm{dL}$ ) and a further fall in levels $(p=0.304)$ at 3 months (mean: $86 \pm 34.08 \mathrm{mg} / \mathrm{dL}$ ). Serum VLDL levels showed a slight decrease at 4 weeks (mean: $35.82 \pm$ $14.79 \mathrm{mg} / \mathrm{dL}$ ) which was not statistically significant $(p=0.790)$ when compared with preoperative levels (mean: $37.23 \pm 15.91 \mathrm{mg} / \mathrm{dL}$ ), and this trend was consistent at 3 months (mean: $32.47 \pm 11.87 \mathrm{mg} / \mathrm{dL}$ ) but again not statistically significant $(p=0.330)$.

\section{Discussion}

Success or failure of any weight-reducing technique is measured by the extent by which body weight is reduced, which is an important parameter of a study. In our study, mean weight loss following bariatric surgery at 4 weeks and 3 months was 8.47 and $22.29 \mathrm{~kg}$, respectively. Out of 17 patients, 16 patients had lost more than $25 \%$ of the excess weight at 3 months following surgery with mean percentage of excess weight loss (\%EWL) at 4 weeks and 3 months being 12.96 and $36.11 \%$, respectively. There was a significant decrease in BMI at 3 months following LSG in our study group with mean BMI at 4 weeks and 3 months being 43.95 and $38.67 \mathrm{~kg} / \mathrm{m}^{2}$ when compared with the preoperative values of $46.67 \mathrm{~kg} / \mathrm{m}^{2}$.

Similar results were found by Alagna et al. ${ }^{22}$ at mean $12 \pm 1$ months postsurgery, the patients showed a significant decrease in weight, from $132.1 \pm 36.9 \mathrm{~kg}$ before surgery to $93.5 \pm 20 \mathrm{~kg}(p<$ $0.0001)$, and BMI, from $47.3 \pm 13.1 \mathrm{~kg} / \mathrm{m}^{2}$ before surgery to $33.5 \pm$ $7 \mathrm{~kg} / \mathrm{m}^{2}(p<0.0001)$.

Omana et al. ${ }^{23}$ found the same results when studied men undergoing surgery lost weight; the $\% E W L$ was $61.48 \%$ (confidence interval: 47.3875-75.5725).

Similarly, mean weight loss observed by Bastounis et al. ${ }^{24}$ in their study was $70 \pm 10 \mathrm{~kg}$ for men. The mean body weight and BMI 12 months after vertical banded gastroplasty were $109 \pm 20.5$ $\mathrm{kg}$ and $34.7 \pm 6.5 \mathrm{~kg} / \mathrm{m}^{2}$, respectively.

Chowbey et al. ${ }^{27}$ reported the Indian experience of 75 patients who underwent LSG for the treatment of morbid obesity. There was a steady rise in $\% E W L$ from $31.2 \%$ at 3 months to $52.3 \%$ at 6 months, $59.13 \%$ at 1 year, and $65 \%$ at 2 years.

An important reason for reduction in $\mathrm{BMI}$ and \%EWL is due to effect of LSG on gastric emptying time. The time required for half of the solid meal to leave the stomach $\left(T_{1 / 2}\right)$ gets significantly altered following LSG, indicating that the stomach empties solid foods rapidly and possibly incompletely processed into the duodenum. Excision of the fundus and absence of receptive relaxation, as well as alterations in the contractile activity in the proximal stomach, are possible explanations for the decreased $T_{1 / 2}$. Laparoscopic sleeve gastrectomy thus induces weight loss by reducing food intake, accelerated gastric emptying, and delivery of nutrients to the small intestine early in the eating cycle which activates small intestine satiety inducing chemoreceptors that modify food ingestion periodicity, inhibit glucagon secretion, and reduce bowel motility and thus reducing appetite and food intake. Postprandial satiety is provoked with significantly less food than preoperatively.

\section{Effect on Biochemical Parameters}

Laparoscopic sleeve gastrectomy has been shown to reduce comorbidities and mortality in patients with morbid obesity and most significantly to ameliorate or resolve type II DM. Much of the improvement has been related to the excess weight loss after surgery. However, some effects appear to be independent from weight loss.

In our study, there was a significant reduction in the levels of $\mathrm{HbA1c}$ at 4 weeks and 3 months postoperatively in diabetic patients with mean $\mathrm{HbA} 1 \mathrm{c}$ at 4 weeks and 3 months being $6.71 \%$ and $5.57 \%$, respectively, when compared with the mean preoperative value of $7.14 \%$.

Todkar et al. ${ }^{25}$ also showed a decrease in HbA1c levels after LSG. This also proves that LSG has a significant role in improvement and remission of diabetes.

The achievement of normoglycemia after bariatric procedures results from multiple changes that occur postoperatively such as control of diet/dietary modifications, decreased plasma ghrelin levels, which, in turn, leads to an increase in maximal capacity of glucose-induced insulin release by the islet cells. Other causes of normoglycemia include loss of weight, reduction of body fat, and the release of gastrointestinal hormones. These hormones further interfere with the function of pancreatic $\beta$-cells (incretins).

Dyslipidemia is a recognized cardiovascular risk factor in obese patients. In our study, there was a significant decrease in serum cholesterol and serum triglycerides levels at 3 months with a significant improvement in serum HDL levels. Serum LDL and serum VLDL levels also showed a decreasing trend, however not statistically significant in this study, citing further follow-up to 1-2 years. Five dyslipidemic patients achieved normal serum cholesterol levels $(<240 \mathrm{mg} / \mathrm{dL}$ ) within 3 months of surgery and thus showed a resolution of dyslipidemia. A study done by Chowbey et al. showed similar results with the resolution of dyslipidemia in $34 \%$ of the patients with a significant decrease in mean cholesterol and LDL levels 6 months after LSG. ${ }^{25}$ Todkar et al. conducted a study on 20 dyslipidemic patients who underwent LSG. Parameters like serum cholesterol, triglycerides, and LDL levels showed a marked increase in HDL levels. ${ }^{26} \mathrm{~A}$ significant decrease in serum cholesterol, LDL, and serum triglyceride levels with an increase in serum HDL levels following sleeve gastrectomy in 130 obese patients undergoing LSG was documented by Hady et al. ${ }^{26}$

\section{Effect on Anthropometric Parameters}

In our study, mean waist circumference of 17 obese patients was $132.94 \pm 13.64 \mathrm{~cm}$ preoperatively. Postoperatively at 4 week, the mean waist circumference decreased to $126.47 \pm 13.24 \mathrm{~cm}$, but waist circumference showed a significant decrease/reduction at 3 months following surgery with the mean values falling down to $106.93 \pm$ $10.84 \mathrm{~cm}$. There was a decrease in waist-to-hip ratio at 4 weeks postoperatively, from mean preoperative value of $1.06 \pm 0.06 \mathrm{~cm}$ to mean values of $1.05 \pm 0.06 \mathrm{~cm}$ and a further significant decrease to $0.98 \pm 0.05 \mathrm{~cm}$ at 3 months, respectively. This signifies that LSG as a sole bariatric procedure decreases the risk of central obesity. In our study, there is no significant change in hip circumference of the patients.

In a study conducted by Hady et al., similar findings were documented in a clinical report which got published in 2012. After 1 year of the surgery, waist circumference in women decreased from $122.8 \pm 18.4 \mathrm{~cm}$ to $89 \pm 8.2 \mathrm{~cm}$ and in men from $134.2 \pm$ $27.6 \mathrm{~cm}$ to $106 \pm 9.66 \mathrm{~cm}^{27}$ 


\section{Conclusion}

Significant weight loss and decrease in anthropometric parameters after bariatric surgery in men results in improved clinical outcomes. Lipid profile and glycemic control also improved in patients over follow-up time. The improvement in these parameters may serve as motivators for obese men considering surgery.

\section{References}

1. Barness LA, Opitz JM, Gilbert-Barness E. Obesity: genetic, molecular and environmental aspects. AM J Med Geneft 2007;143(24): 3016-3034. DOI: 10.1002/ajmg.a.32035.

2. Saber AA, El-Ghazaly TH. Early experience with single-access transumbilical adjustable laparoscopic gastric banding. Obes Surg 2009;19(10):1442-1446. DOI: 10.1007/s11695-009-9905-8.

3. NIH consensus statement covers treatment of obesity. Am Fam Physician 1991;44(1):305-306.

4. Sjöström L, Narbro K, Sjöström CD, et al. Effects of bariatric surgery on mortality in Swedish obese subjects. N Engl J Med 2007;357(8): 741-752. DOI: 10.1056/NEJMoa066254.

5. Buchwald H, Avidor Y, Braunwald E, et al. Bariatric surgery: a systematic review and meta-analysis. JAMA 2004;292(14):1724-1737. DOI: 10.1001/jama.292.14.1724.

6. Buchwald H, Oien DM. Metabolic/bariatric surgery worldwide 2008. Obes Surg 2009 19(12):1605-1611. DOI: 10.1007/s11695-009-0014-5.

7. Malone M. Medications associated with weight gain. Ann Pharmacother 2005;39(12):2046-2055. DOI: 10.1345/aph.1G333.

8. Ness-Abramof R, Apovian CM. Drug-induced weight gain. Drugs Today (Barc) 2005;41(8):547-555.

9. Gurevich-Panigrahi T, Panigrahi S, Wiechec E, et al. Obesity: pathophysiology and clinical management. Curr Med Chem 2009;16(4):506-521. DOI: 10.2174/092986709787315568.

10. Korner J, Woods SC, Woodworth KA. Regulation of energy homeostasis and health consequences in obesity. Am J Med 2009;122(4 suppl 1):S12-S18. DOI: 10.1016/j.amjmed.2009.01.003.

11. Brolin RE. Update: $\mathrm{NIH}$ consensus conference. Gastrointestinal surgery for severe obesity. Nutrition 1996;12(6):403-404. DOI: 10.1016/s08999007(96)00154-2.

12. Malt RA, Kral JG. Surgery for obesity. In: Morris PJ, Malt RA. Oxford Textbook of Surgery. New York: Oxford University Press; 1994.

13. Lubrano C, Mariani S, Badiali M, et al. Metabolic or bariatric surgery? long-term effects of malabsorptive vs restrictive bariatric techniques on body composition and cardiometabolic risk factors. Int J Obes (Lond) 2010;34(9):1404-1414. DOI: 10.1038/ijo.2010.54.

14. Weiss R, Appelbaum L, Schweiger $C$, et al. Shortterm dynamics and metabolic impact of abdominal fat depots after bariatric surgery. Diabetes Care 2009;32(10):1910-1915. DOI: 10.2337/dc090943.

15. DeMaria EJ. Bariatric surgery for morbid obesity. N Engl J Med 2007;356(21):2176-2183. DOI: 10.1056/NEJMct067019.

16. Brethauer SA, Hammel JP, Schauer PR. Systematic review of sleeve gastrectomy as staging and primary bariatric procedure. Surg Obes Relat Dis 2009;5(4):469-475. DOI: 10.1016/j.soard.2009.05.011.

17. Demaria EJ, Winegar DA, Pate VW, et al. Early postoperative outcomes of metabolic surgery to treat diabetes from sites participating in the ASMBS bariatric surgery center of excellence program as reported in the bariatric outcomes longitudinal database. Ann Surg 2010;252(3):559-566. DOI: 10.1097/SLA.0b013e3181f2aed0.

18. Mechanick JI, Kushner RF, Sugerman HJ, et al. American association of Clinical Endocrinologists, the Obesity Society, and American Society for Metabolic \& Bariatric Surgery medical guidelines for clinical practice for the perioperative nutritional, metabolic, and nonsurgical support of the bariatric surgery patient. Obesity (Silver Spring) 2009;17(Suppl 1):S1-S70. DOI: 10.1038/oby.2009.28.

19. Birkmeyer NJ, Wei Y, Goldfaden A, et al. Characteristics of hospitals performing bariatric surgery. JAMA 2006;295(3):282-284. DOI: 10.1001/jama.295.3.282-c.

20. Buchwald H, Estok R, Fahrbach K, et al. Trends in mortality in bariatric surgery: a systematic review and meta-analysis. Surgery 2007;142(4):621-632. DOI: 10.1016/j.surg.2007.07.018.

21. Kelly JJ, Shikora S, Jones DB, et al. Best practice updates for surgical care in weight loss surgery. Obesity (Silver Spring) 2009;17(5):863-870. DOI: $10.1038 /$ oby.2008.570.

22. Alagna S, Cossu ML, Gallo P, et al. Biliopancreatic diversion: long-term effects on gonadal function in severely obese men. Surg Obes Relat Dis 2006;2(2):82-86. DOI: 10.1016/j.soard.2006.01.005.

23. Omana JJ, Tamler R, Strohmayer E, et al. Sex hormones in men undergoing bariatric surgery. J Am Coll Surg 2009;209(3):S22-S23. DOI: 10.1016/j.jamcollsurg.2009.06.042.

24. Bastounis EA, Karayiannakis AJ, Syrigos K, et al. Sex hormone changes in morbidly obese patients after vertical banded gastroplasty. Eur Surg Res 1998;30(1):43-47. DOI: 10.1159/000008556.

25. Todkar JS, Shah SS, Shah PS, et al. Long-term effects of laparoscopic sleeve gastrectomy in morbidly obese subjects with type 2 diabetes mellitus. Surg Obes Relat Dis 2010;6(2):142-145. DOI: 10.1016/ j.soard.2009.06.008.

26. Hady H, Dadan J, Luba M. The influence of laparoscopic sleeve gastrectomy on metabolic syndrome parameters in obese patients in own material. Obes Surg 2012;22(1):13-22. DOI: 10.1007/s11695011-0530-y.

27. Chowbey PK, Dhawan K, Khullar R. Laparoscopic sleeve gastrectomy: an Indian experience — surgical technique and early result. Obes Surg 2010;20(10):1340-1347. DOI: 10.1007/s11695-009-9973-9. 